\title{
Resuscitation Endpoints in Trauma
}

\section{SUMMARY}

LENA M. NAPOLITANO, MD, FACS, FCCP, FCCM

Professor OF SURGERY

University of Michigan SCHOOL OF Medicine Ann Arbor, Michigan, USA
Fluid and blood resuscitation is the mainstay of therapy for the treatment of hemorrhagic shock, whether due to trauma or other etiology. Cessation of hemorrhage with rapid hemostatic techniques is the first priority in the treatment of traumatic hemorrhagic shock, with concomitant fluid resuscitation with blood and crystalloids to maintain perfusion and organ function. "Hypotensive" or "low-volume" resuscitation has become increasingly accepted in the prehospital resuscitation phase of trauma, prior to definitive hemorrhage control, since aggressive fluid resuscitation may increase bleeding. Resuscitation after hemorrhage control is focused on restoration of tissue oxygenation. E fforts to optimize resuscitation have used "resuscitation endpoints" as markers of adequacy of resuscitation. The resuscitation endpoints that have been evaluated include both global (restoration of blood pressure, heart rate and urine output, lactate, base deficit, mixed venous oxygen saturation, ventricular end-diastolic volume) and regional (gastric tonometry, near-infrared spectroscopy for measurement of muscle tissue oxygen saturation) measures. This review critically evaluates the evidence regarding the use of resuscitation endpoints in trauma. 
Hemorrhagic shock due to trauma is associated with a high mortality rate. Early mortality is related to an inability to promptly control hemorrhage, and late mortality is related to reperfusion injury, organ failure, and septic complications. In a study of 208 patients with hemorrhagic shock (defined as systolic blood pressure $[S B P] \leq 90 \mathrm{~mm} \mathrm{Hg}$ in the field or emergency department) cared for at a Level I trauma center, $54 \%$ died. ${ }^{1}$ In this study, 31\% died in 2 hours, $12 \%$ died between 2 and 24 hours, and $11 \%$ died after 24 hours. Among those who survived > 24 hours, 39\% developed infection and 24\% developed organ failure. Increasing volume of crystalloid in the first 24 hours was strongly associated with increased mortality $(p=0.00001)$. This study clearly documented that hemorrhageinduced hypotension in trauma patients is predictive of high mortality (54\%). We also recognize, however, that trauma patients may have different degrees of hemorrhagic shock. Some trauma victims have transient hypotension indicative of mild hemorrhagic shock that is easily correctable with resuscitation. Others have persistent hypotension due to severe hemorrhagic shock, despite aggressive resuscitation, and associated with multiple organ dysfunction and very high mortality rates.

Fluid resuscitation is the mainstay of therapy for the treatment of hemorrhagic shock that results in end-organ hypoperfusion. Efforts to improve outcome from hemorrhagic shock in trauma have therefore focused on adequacy of resuscitation. Traditionally, the return to normal blood pressure, heart rate, and urine output has been used as the endpoint of resuscitation. A number of studies have documented, however, that the use of these endpoints may leave a substantial number of patients (up to 50 to $85 \%$ in some series) in "compensated" shock, which if it persists may ultimately lead to the death of the patient. Based on this finding, other resuscitation endpoints have been evaluated, including oxygen transport variables (mixed venous oxygen saturation, oxygen delivery, and oxygen consumption), lactate, base deficit, and gastric intramucosal $\mathrm{pH}$. Some have advocated that the goal should be to correct these resuscitation endpoints or markers of tissue perfusion to normal within the initial 24 hours after injury. ${ }^{2}$ There is concern that attempts to do this may result in over-resuscitation, and others have recommended more modest resuscitation. Furthermore, the determination of adequate intravascular volume or preload remains difficult in critically ill and injured patients, and the use of pulmonary capillary wedge pressure, mixed venous oxygen saturation and oxygen delivery as endpoints for resuscitation are problematic. ${ }^{3} \mathrm{~N}$ ormalization of tissue perfusion and oxygenation is the goal of fluid resuscitation in shock, but the markers currently available are not accurate measures of tissue perfusion and oxygenation.

Vigorous intravenous fluid resuscitation has long been the standard management of hemorrhagic shock in trauma. There is now, however, sufficient evidence for this position to be reviewed. Hypotensive or delayed resuscitation has been postulated as a means by which the mortality associated with hemorrhagic shock can be reduced. It has been suggested that overresuscitation with intravenous fluids may worsen hemorrhage prior to definitive hemorrhage control. The adverse effects of "conventional" resuscitation have been critically examined and alternative resuscitation strategies, including hypotensive, delayed or more moderate fluid resuscitation have been investigated. This review examines the use of resuscitation endpoints in trauma, and resuscitation strategies in trauma care.

\section{Prehospital Resuscitation}

Significant controversy still exists regarding whether fluid resuscitation after injury should be initiated in the prehospital setting. ${ }^{4}$ Fluid resuscitation may be detrimental when given before bleeding is controlled in patients with trauma if it results in increased mean arterial pressure and increased bleeding at the site of injury. Animal studies have documented that total volume of blood loss in trauma is related to mean arterial pressure, and that hemorrhage ceased spontaneously significantly earlier in both delayed and no fluid resuscitation groups compared to immediate fluid resuscitation. Early aggressive fluid resuscitation exacerbated total hemorrhage volume and delayed fluid resuscitation resulted in decreased hemorrhage and the best hemodynamic response to shock. ${ }^{5}$ Similarly, aggressive resuscitation in a rat uncontrolled hemorrhage model due to aortic laceration was associated with a high mortality rate, as was the no fluid resuscitation group. ${ }^{6}$ The groups that received moderate resuscitation (mean $\mathrm{BP}, 80 \mathrm{~mm} \mathrm{Hg}$ with lactated Ringer's or $40 \mathrm{~mm} \mathrm{Hg}$ with $7.5 \%$ hypertonic saline and $6 \%$ hetastarch) had the highest survival rates in this study.

The appropriate resuscitation endpoint in the prehospital setting is unclear. The available evidence does not clearly support any single approach. In patients who are hemodynamically stable, maintenance of a SBP of 90 or a palpable radial pulse may be adequate in some trauma victims, but may be inadequate in others, such as those with severe traumatic brain injury. Few clinical studies in this area of investigation are published.

\section{Hypotensive or Delayed Resuscitation}

During uncontrolled hemorrhagic shock in acute animal models, attempts to achieve normotension with intravenous fluid resuscitation were associated with further bleeding and higher acute mortality. The development of animal models of uncontrolled hemorrhagic shock that mimicked the clinical situation required a "prehospital" and "hospital" phase of resuscitation to study outcome. ${ }^{7}$ A number of preclinical animal studies using this uncontrolled hemorrhagic shock model with two phases of resuscitation have documented the beneficial effects of delayed fluid resuscitation until definitive hemorrhage control. ${ }^{8-10}$ But many 
of these animal models differ with regard to the site of injury (solid organ, arterial injury), duration of hemorrhage, and type of fluids infused for resuscitation (crystalloid, hypertonic saline, blood, colloids). Other animal studies document the potential benefits of small-volume "hypotensive" resuscitation, maintaining a mean arterial pressure of $40 \mathrm{~mm} \mathrm{Hg}$ during the prehospital phase with limited resuscitation, on outcome in a rat uncontrolled hemorrhagic shock model. .11, $^{12}$

The landmark study by Bickell and colleagues ${ }^{13}$ examined the effect of delaying fluid resuscitation until the time of surgical intervention in hypotensive patients with penetrating torso injuries in an urban setting with a single centralized system of prehospital emergency care and a single receiving facility for patients with major trauma. This prospective trial randomized patients ( $n=598$ ) with SBP $\leq 90 \mathrm{~mm} \mathrm{Hg}$ to immediate resuscitation (standard prehospital fluid resuscitation) and delayed resuscitation (intravenous cannulation but no prehospital fluid resuscitation). Delayed resuscitation was associated with improved hospital survival ( $70 \%$ vs. $62 \%, p=0.04$ ), shorter duration of hospitalization, with no increase in complications ( $23 \%$ vs. $30 \%, p=0.08$ ). It should be noted, however, that the study patients were young with a mean age of 31 years, with short transport times in this urban setting.

A similar prehospital study in blunt trauma patients $(n=1309)$ was completed in the United Kingdom, randomizing patients to immediate fluids vs. no prehospital fluids. No outcome differences were identified between groups, but protocol compliance was poor, with only $31 \%$ of the immediate fluid group and $20 \%$ of the no fluid group receiving prehospital fluid resuscitation. ${ }^{14} \mathrm{~A}$ retrospective case-matched control study compared blunt trauma victims $(n=150)$ with $\mathrm{SBP} \leq 90 \mathrm{~mm}$ $\mathrm{Hg}$ who received $>500 \mathrm{~mL}$ prehospital fluids to those who did not receive any prehospital fluids, matched by Injury Severity Score (ISS) and systolic blood pressure on scene. Although trauma patients that received prehospital fluid had an increased SBP on arrival, there were no significant differences in hospital survival or length of stay. ${ }^{15}$

A recent consensus statement was published regarding prehospital fluid resuscitation by the Faculty of Pre-Hospital Care of the Royal College of Surgeons, Edinburgh. ${ }^{16}$ This group recommended that intravenous cannulation should be attempted en route when possible, and that transfer should not be delayed by attempts to obtain intravenous access, except for entrapped patients who require intravenous cannulation at the scene. Fluid resuscitation is recommended to be titrated in boluses of $250 \mathrm{~mL}$ to maintain the presence of a radial arterial pulse, implying a SBP of approximately $90 \mathrm{~mm} \mathrm{Hg}$. Similarly, Rhee and colleagues recommend a delayed strategy for the initial resuscitation of combat casualties (Figure 1). ${ }^{17}$

\section{Figure 1}

Algorithm for the Initial Resuscitation of Trauma Victims

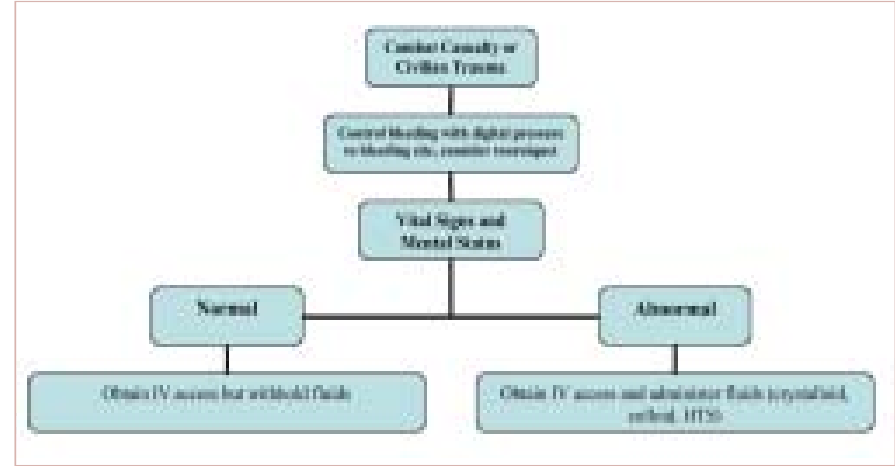

Adapted from Rhee et al. ${ }^{11}$

\section{In-Hospital Resuscitation}

The standard in-hospital resuscitation strategy for the treatment of trauma patients with hemorrhagic shock is the rapid infusion of 2 liters of crystalloid, followed by prompt blood transfusion with uncrossmatched blood if persistent hypotension or tachycardia, advocated by the Advanced Trauma Life Support guidelines. Additional crystalloid resuscitation and blood transfusion is based on clinical criteria and physiologic response to individual fluid boluses.

The potential benefits of limited or hypotensive fluid resuscitation prior to definitive hemorrhage control identified in the prehospital setting have al so been considered for the hospital setting. Data derived from preclinical animal studies strongly suggest that hypotensive resuscitation may be preferable for the trauma victim with the potential for ongoing uncontrolled hemorrhage. Hypotensive resuscitation provides a mechanism of avoiding the detrimental effects associated with early aggressive resuscitation, while maintaining a level of tissue perfusion that, although decreased from the normal physiologic range, is adequate and tolerated for short periods. ${ }^{18}$ To date, however, no large randomized clinical trials have yet confirmed these animal studies.

One recent study examined different fluid resuscitation strategies in actively hemorrhaging trauma patients, to determine if fluid resuscitation titrated to a lower blood pressure during the period of active hemorrhage would result in improved survival. Patients were eligible for inclusion if they presented directly from the scene of a traumatic injury, had evidence of ongoing hemorrhage, and had a SBP $<90 \mathrm{~mm} \mathrm{Hg}$ recorded at least once within the first hour after injury. Patients were excluded if they were pregnant, had a central nervous system injury impairing their level of consciousness or motor function, were older than 55, or had a previous medical history of diabetes or coronary artery disease. Trauma patients in hemorrhagic shock ( $n=110$ ) were randomized on hospital admission to a target SBP > 100 mm Hg ("conventional" group) or 70 mm Hg ("Iow" 
group) maintained with fluid resuscitation until definitive hemostasis was achieved. ${ }^{19}$ Blood pressure below the target level was treated with administration of crystalloid or blood products, as appropriate to elevate the SBP to the target level while maintaining a hematocrit of at least $25 \%$. Sustained SBP above the target level was managed by restriction of fluids and administration of appropriate doses of anesthetic or analgesic medication. The mean age of the study cohort was 31 years and penetrating trauma victims comprised $51 \%$ of the study cohort. No difference in survival was demonstrated, with overall survival being $92.7 \%$. Potential reasons for lack of outcome difference in this study include the following: (1) inclusion of both blunt and penetrating trauma victims; (2) SBP higher than study design in the hypotensive resuscitation group during the study period (100 mm Hg vs. $114 \mathrm{~mm} \mathrm{Hg}$ in control group, $p<0.001$ ); and (3) improved hemorrhage control methods resulting in lower overall mortality.

Large randomized clinical trials are necessary to confirm the potential benefits of hypotensive resuscitation until definitive hemorrhage control. Specific patient groups that may have adverse outcome related to hypotensive resuscitation include the elderly and those with severe traumatic brain injury. O ptimal resuscitation methods in these patient groups must be further examined. Future research should focus on developing resuscitation methods that may actually enhance tissue perfusion during limited or hypotensive resuscitation and therefore offset its potential detrimental effects.

\section{Lactate and Base Deficit As Resuscitation Endpoints}

It is clearly recognized that elevated lactate and base deficit on hospital admission after trauma reflect impaired tissue perfusion of the shock state and adverse outcome. The time to normalization of arterial lactate concentration has also been associated with outcome, with longer time to normalization associated with increased mortality. In a study of 95 critically ill patients requiring hemodynamic monitoring, patients that normalized lactate in $<24$ hours had the lowest mortality rate (3.9\%) compared to $13.3 \%$ mortality in those normalizing in $24-48$ hours and $42.5 \%$ mortality in those normalizing in 48-96 hours. Patients that did not achieve a normal lactate level had a $100 \%$ hospital mortality rate. Multivariate analysis confirmed that time to lactate clearance was an independent predictor of survival. This study clearly documented that prolongation of lactate clearance was associated with increased mortality. ${ }^{20}$
A more recent study $(n=137)$ confirmed that elevated initial and 24-hour lactate levels and 24-hour base deficit were associated with increased mortality. In this study, mortality was $10 \%$ if lactate normalized within 2 hours, compared with mortality rates of $24 \%$ for $>48$ hours and $67 \%$ if lactate failed to normalize. Importantly, physical status at discharge was also related to initial lactate as well as to lactate clearance time. ${ }^{21}$

The Eastern Association for Surgery of Trauma Clinical Practice Guideline on Endpoints of Resuscitation published a Level 1 recommendation that initial base deficit, lactate level or gastric $\mathrm{pHi}$ from tonometry can be used to stratify patients with regard to the need for ongoing fluid resuscitation (Table 1 ). ${ }^{22}$ Although these studies clearly document that arterial lactate is associated
Recommendations of the EAST Clinical Practice Guideline: Endpoints of Resuscitation

\section{A. Recommendations Regarding Stratifying Physiologic Derangement}

Level 1 1. Standard hemodynamic parameters do not adequately quantify the degree of physiologic derangement in trauma patients. The initial base deficit, lactate level, or gastric pHi can be used to stratify patients with regard to the need for ongoing fluid resuscitation, including packed red blood cells and other blood products, and the risks of multiple organ dysfunction syndrome and death.

2. The ability of a patient to attain supranormal oxygen delivery parameters correlates with an improved chance for survival.

Level 2 1. The time to normalization of base deficit, lactate and pHi is predictive of survival.

2. Persistently high base deficit or low pHi (or worsening of these parameters) may be an early indicator of complications, e.g., ongoing hemorrhage or abdominal compartment syndrome.

3. The predictive value of the base deficit may be limited by ethanol intoxication or a hyperchloremic metabolic acidosis as well as administration of sodium bicarbonate.

Level 3 1. Right ventricular end diastolic volume index (RVEDVI) measurement may be a better indicator of adequate volume resuscitation (preload) than central venous pressure or pulmonary capillary wedge pressure (PCWP).

2. Measurements of tissue (subcutaneous or muscle) oxygen and/or carbon dioxide levels may identify patients who require additional resuscitation and are at risk for multiple organ dysfunction syndrome and death.

3. Serum bicarbonate levels may be substituted for base deficit levels.

\section{B. Recommendations Regarding Improved Patient Outcomes}

Level 1 There is insufficient data to formulate a Level 1 recommendation.

Level 2 1. During resuscitation, attempts should be made to increase oxygen delivery to normalize base deficit, lactate or pHi during the first 24 hours. The optimal algorithms for fluid resuscitation, blood product replacement, and the use of inotropes and/or vasopressors have not been determined.

Adapted from Tisherman et al. ${ }^{16}$; available at www.east.org. 
with adverse outcome in trauma, no prospective studies have investigated if standardized methods for trauma resuscitation would improve lactate clearance time, lactate and base deficit normalization and improve outcomes.

\section{Oxygen Delivery and Consumption} As Resuscitation Endpoints in Trauma

Shoemaker and colleagues performed the first prospective evaluation of supranormal values of cardiac index $(\mathrm{Cl})$, oxygen deliver index $\left(\mathrm{DO}_{2} \mathrm{l}\right)$ and oxygen consumption index $\left(\mathrm{VO}_{2} \mathrm{I}\right)$ as resuscitation goals to improve outcome in severely traumatized patients. ${ }^{23}$ The protocol resuscitation goals were $\mathrm{Cl} \geq 4.5 \mathrm{~L} / \mathrm{min} / \mathrm{m}^{2}$, $\mathrm{DO}_{2} \mathrm{I} \geq 670 \mathrm{~mL} / \mathrm{min} / \mathrm{m}^{2}$, and $\mathrm{VO}_{2} \mathrm{I} \geq 166 \mathrm{~mL} / \mathrm{min} / \mathrm{m}^{2}$ within 24 hours of admission. The control resuscitation goals were normal vital signs, urine output, and central venous pressure. The 50 protocol patients had a significantly lower mortality (9 of 50, $18 \%$ vs. 24 of $65,37 \%$ ) and fewer organ failures per patient $(0.74 \pm 0.28$ vs. $1.62 \pm 0.45)$ than did the 75 control patients. This study documented that increased $\mathrm{Cl}, \mathrm{DO}_{2} \mathrm{l}$, and $\mathrm{VO}_{2} \mathrm{I}$ seen in survivors of severe trauma are primary compensations that are associated with improved survival.

Other similar studies failed to achieve improved survival in patients randomized to supranormal oxygen consumption and delivery as endpoints for resuscitation in trauma. Durham et al. reported that no difference was found in the incidence of organ failure or death in patients resuscitated based on oxygen transport parameters compared to conventional parameters. ${ }^{24}$ These data suggested that given adequate volume resuscitation, oxygenbased parameters are more useful as predictors of outcome than as endpoints for resuscitation. Similarly, Velmahos and colleagues, in a prospective randomized study of 75 severely injured patients, documented no difference in outcome in patients randomized to "optimal" or supranormal resuscitation. ${ }^{25}$ O ptimal values were reached intentionally by $70 \%$ of the optimal patients and spontaneously by $40 \%$ of the control patients. There was no difference in rates of death ( $15 \%$ optimal vs. $11 \%$ control), organ failure, sepsis, or the length of intensive care unit or hospital stay between the two groups. Patients from both groups who achieved optimal values had better outcomes than patients who did not. The death rate was $0 \%$ among patients who achieved optimal values compared with $30 \%$ among patients who did not. Age younger than 40 years was the only independent predictive factor of the ability to reach optimal values. This study documented that severely injured patients who can achieve optimal hemodynamic values are more likely to survive than those who cannot, regardless of the resuscitation technique. In this study, attempts at early optimization did not improve the outcome of severely injured patients.

A recent meta-analysis of hemodynamic optimization in medical and surgical high-risk patients, including trauma victims, documented that "early" optimization (defined as 8-12 hours postoperatively or before organ failure) and increased severity of illness were independent characteristics of improved outcome in the "optimal" resuscitation groups. Review of 21 randomized controlled trials with various approaches to treatment revealed statistically significant mortality reductions, with hemodynamic optimization, when patients with acute critical illness were treated early to achieve optimal goals before the development of organ failure, when there were control group mortalities of $>20 \%$ and when therapy produced differences in oxygen delivery between the control and protocol groups. ${ }^{26}$

Most recently, a report by McKinley and colleagues documented that trauma resuscitation using a computerized bedside protocol for the standardized resuscitation of trauma victims using a $\mathrm{DO}_{2} \mathrm{I}$ goal endpoint of $600 \mathrm{vs} .500 \mathrm{~mL} / \mathrm{min} / \mathrm{m}^{2}$ for the first 24 hours using primarily blood and volume loading resulted in no outcome difference. ${ }^{27}$ Importantly, both crystalloid and blood transfusion volume were reduced in the lower $\mathrm{DO}_{2} \mathrm{I}$ group. Throughout the 24-hour ICU process, the $\mathrm{DO}_{2} \mathrm{I} \geq 500$ cohort received less lactated Ringer's volume than the $\mathrm{DO}_{2} \mathrm{I} \geq 600$ cohort (total of $8 \pm 1$ vs. $12 \pm 2 \mathrm{~L} ; \mathrm{p}<0.05$ ) and received less blood transfusion (total of $3 \pm 1$ vs. $5 \pm 1$ units of packed red blood cells).

\section{Confounding Variables in Resuscitation Studies}

\section{Age}

There are a number of potential confounding variables that may limit the results of prior resuscitation studies in trauma, particularly with regard to optimization of resuscitation endpoints in trauma. It is clearly recognized that age is a significant confounding variable, since age has been identified as an independent predictor of adverse outcome and increased mortality in trauma. In a recent study of 26,237 blunt trauma patients, being elderly (age $\geq 65$ years, $n=7,117$ ) was associated with a nearly twofold increased mortality risk (odds ratio, 1.87; confidence interval, 1.60-2.18; $p>0.001$ ) on logistic regression analysis after stratification by injury severity score, revised trauma score and other preexisting comorbidities. ${ }^{28}$ Similarly, another study documented that prolonged occult hypoperfusion (defined as serum lactic acid $>2.4 \mathrm{mmol} / \mathrm{L}$ persisting for $>12$ hours) was associated with increased mortality in elderly patients (age $>55$ years) compared to the younger cohort. ${ }^{29} \mathrm{This}$ study documented that elderly patients are a group that have potentially less physiologic reserve, and emphasizes the importance of stratification for age in all resuscitation studies in trauma. Attempts to optimize oxygen delivery in the elderly patient population are commonly unsuccessful, have not been associated with improved outcome, and should only be cautiously implemented in the elderly trauma victim. ${ }^{30}$ 


\section{Type of Fluid for Resuscitation}

Another potential confounding variable in resuscitation studies is the use of different fluids for resuscitation in trauma, including normal saline, lactated Ringer's solution, hypertonic saline, and other artificial colloids. In 1999, the United States Navy requested the Institute of Medicine to review the evidence on fluid resuscitation for combat casualties. ${ }^{31}$ This report documented the evidence regarding potential adverse effects (immune activation and induction of cellular injury) of some fluids used in the resuscitation of trauma victims. This report made a final recommendation to use $7.5 \%$ hypertonic saline (up to $500 \mathrm{~mL}$ ) for the treatment of battlefield casualties in shock. Since hypertonic saline can provide the same hemodynamic response as isotonic crystalloids with ap proximately one-tenth the volume and weight, it makes sense to utilize this in the combat and military arena. It has not yet been determined whether this is the ideal resuscitation fluid in the non-combat trauma environment.

A recent meta-analysis of individual patient data from a multicenter study of hypertonic saline dextran (HSD) examined its efficacy in patients $(n=230)$ with penetrating injuries to the torso..$^{32}$ The administration of HSD may cause an immediate and sustained increase in blood pressure that could contribute to an increase in bleeding in the presence of uncontrolled hemorrhage. Of the 120 patients treated with HSD, $82.5 \%$ survived compared with $75.5 \%$ for 110 standard of care patients $(p=0.19)$. Sixtyeight percent ( $n=157$ ) of these patients required surgery. HSD treatment $(n=84)$ in this population improved survival, $84.5 \%$ compared with $67.1 \%$ with SOC $(n=73)(p=0.01)$. HSD resulted in an increase in blood pressure and a reduction in hematocrit, with no differences noted in fluid requirements or indices of clotting. These retrospective data suggest that in patients with penetrating injuries to the torso that result in hypotension, initial fluid resuscitation with HSD may be beneficial in improving survival, especially if surgery is subsequently required.

Most recently, prehospital hypertonic saline was studied in Australia for the resuscitation of patients with traumatic brain injury. ${ }^{33}$ Patients who were comatose (GCS $<9$ ) and hypotensive (SBP $<100 \mathrm{~mm} \mathrm{Hg}, \mathrm{n}=229$ ) were randomized to prehospital resuscitation with $250 \mathrm{~mL}$ of intravenous hypertonic saline (7.5\%) or $250 \mathrm{~mL}$ of Ringer's lactate. No differences in hospital and 6-month survival rates were identified, and no differences in postinjury neurological function were identified in this prospective, randomized, double-blind study.

$\mathrm{N}$ ewer synthetic colloids are under active investigation in Europe.$^{34} \mathrm{~A}$ new hydroxyethyl starch with a molecular weight of $130,000 \mathrm{D}$ is without significant influence on coagulation and may reduce the inflammatory response of monocytes and endothelial cells compared to crystalloid-based fluid resuscitation. ${ }^{35,36}$ Additional studies regarding the use of this new hydroxyethyl starch in the acute resuscitation of trauma victims are warranted.

The ideal resuscitation fluid for the trauma patient would be capable of reversing tissue hyp oxia in critical organ beds. A recent study in a porcine hemorrhagic shock model compared smallvolume resuscitation with a hemoglobin-based oxygen carrier (HBOC-201, Biopure Corp., Cambridge, MA, USA; $6 \mathrm{~mL} / \mathrm{kg}$ ) to lactated Ringer's solution (12 mL/kg) or hypertonic saline dextran (HSD, $4 \mathrm{~mL} / \mathrm{kg}$ ) in restoring baseline tissue oxygen tension levels in selected tissue beds after hemorrhagic shock. ${ }^{37}$ There were no significant differences in measured liver or muscle tissue oxygenation values after resuscitation with any of the three solutions in this model of hemorrhagic shock. When comparing the hemodynamic effects of resuscitation, the cardiac output was increased from shock values in all three animal groups with resuscitation, but was significantly higher in the animals resuscitated with HSD. HBOC-201 was most effective in both restoring and sustaining MAP and systolic blood pressure $(p<0.01)$. This study also documented that there was excellent correlation between liver and deltoid muscle tissue oxygen values $(r=0.8, p<0.0001)$, and thus deltoid muscle $\mathrm{PO}_{2}$ may serve as an index of the adequacy of resuscitation in critical tissue beds.

\section{Measures of Adequate Preload in Trauma As Resuscitation Endpoints}

Since hemorrhage and hypovolemia are the underlying etiology of shock in the vast majority of trauma victims, determination of adequacy of fluid resuscitation by measurement of preload is necessary. A number of studies have documented the limitations of central venous pressure and pulmonary capillary wedge pressure as measures of preload in critically ill trauma victims, due to altered cardiac compliance, and increased intrathoracic and intra-abdominal pressure. In patients at risk for increased intra-abdominal pressure and abdominal compartment syndrome, it has been documented that pulmonary capillary wedge pressure parallels the intra-abdominal pressure and is not an accurate measure of preload. Furthermore, the right ventricular end-diastolic volume index (RVEDVI) correlated better with cardiac index than wedge pressure. Pulmonary capillary wedge pressure is therefore an unreliable index of preload in patients with increased intra-abdominal pressure. ${ }^{38}$ Despite this, some groups still utilize pulmonary capillary wedge pressure and cardiac index to guide preload-directed resuscitation in trauma. ${ }^{39}$

While RVEDVI has been shown to be a better indicator of preload than cardiac filling pressures, optimal values during resuscitation from trauma are unknown, and a volumetric pulmonary artery catheter is required. A recent report documented that optimal individual RVEDVI in 19 critically injured patients could be determined based on measurements 
of ventricular compliance after sequential volume loading with fluid boluses of $500-1000 \mathrm{ml}$. In this standardized resuscitation protocol, the target RVEDVI was $\geq 120 \mathrm{~mL} / \mathrm{m}^{2} .{ }^{40}$

Chang and colleagues have documented that maintaining left ventricular power output (LVP $=$ cardiac index $x$ [mean arterial pressure - central venous pressure]) $>320 \mathrm{~mm} \mathrm{Hg} \mathrm{x} \mathrm{L/min/m²}$ during resuscitation was associated with faster resolution of acidosis and survival after posttraumatic shock in a retrospective study. A subsequent prospective study of 20 patients was compared with 39 control patients who were resuscitated in a prior prospective study based on oxygen transport criteria. Patients with optimized LVP cleared base deficit more quickly, had lower base deficit at 24 hours post-injury, and had decreased organ dysfunction, but survival was not different between groups. ${ }^{41}$

\section{Experimental Resuscitation Endpoints}

\section{Near-Infrared Spectroscopy}

Current measurements that are used to determine oxygen delivery to tissues indicate total body perfusion, and regional alterations in oxygen delivery are not detected. N ear-infrared spectroscopy (NIRS) can be used to monitor regional tissue oxygenation during hemorrhagic shock and resuscitation. Initial animal studies confirmed that regional tissue oxygenation measured by NIRS demonstrated a very high correlation with global oxygen delivery. ${ }^{42}$

Noninvasive hindlimb muscle tissue oxygenation measured by NIRS was determined to be more reliable than invasive oxygenation variables (mixed venous oxygen saturation, arterial lactate, arterial base deficit) in a swine hemorrhagic shock model, and may represent an improved method to gauge the severity of shock and the adequacy of fluid resuscitation after trauma. ${ }^{43}$ Recently, NIRS has also been utilized as an endpoint for resuscitation in a computer-controlled closed-loop resuscitation model using invasive cardiac output or noninvasive skeletal muscle oxygen saturation via NIRS in sheep. This study documented that closed-loop resuscitation with either cardiac output or skeletal muscle NIRS as resuscitation endpoints was effective in the resuscitation of severe uncontrolled hemorrhagic shock in a sheep model ${ }^{44}$ Interestingly, the NIRS endpoint resuscitation group required only half as much fluid through 4 hours of resuscitation as the cardiac output endpoint group.

Cohn and colleagues recently reported their results of the use of NIRS in adult human trauma patients for continuous noninvasive determination of tissue oxygen saturation $\left(\mathrm{StO}_{2}\right)$ in muscle. ${ }^{45}$ The use of muscle NIRS applied to the thenar eminence was evaluated in 707 volunteers and 145 trauma patients which were stratified into groups based on no evidence of shock, mild shock (SBP >90, heart rate 100-140, base deficit -3 to -5 ) or moderate shock (SBP $<90$, heart rate $>140$, base deficit $>-5$ ). These investigators established a normal $\mathrm{StO}_{2}$ range with a mean of $86.6 \pm 6.4 \%$ in normal volunteers, and a significant reduction was noted in trauma patients with moderate shock, to a mean of $58.6 \pm 28.4 \%$. This emerging noninvasive technology warrants further careful study in trauma resuscitation.

Other Non-invasive Methods (Bioimpedance, Esophageal Doppler, Transcutaneous Monitoring)

N oninvasive methods of hemodynamic monitoring in trauma patients would be a significant advance, since at present the placement of a pulmonary artery catheter is required to obtain these serial measurements during resuscitation. Noninvasive methods that have been investigated include thoracic bioelectric impedance that estimates cardiac output continuously, esophageal Doppler methods, Pulse contour cardiac output system (PiCCO), and transcutaneous oxygen and carbon dioxide sensors.

Cardiac output estimations by bioimpedance and standard thermodilution technique were well correlated in 38 severely injured blunt trauma patients $(r=0.91) .^{46}$ This study suggested that noninvasive monitoring may be used for early detection and correction of post-traumatic circulatory deficits. The PiCCO monitor combines arterial thermodilution with analysis of the pulse waveform, providing a series of hemodynamic parameters useful for managing the critically ill trauma patient. With the PiCCO system it is possible to measure intrathoracic blood volume, extravascular lung water and cardiac function index (CFI). These parameters are of interest as they are considered to be the most specific measures of cardiac preload, pulmonary edema and contractility and a global indicator of cardiac performance. A recent study compared three methods, including PiCCO, esophageal Doppler and pulmonary artery catheter monitoring in critically ill patients. ${ }^{47}$

Noninvasive transcutaneous oxygen $\left(\mathrm{PtcO}_{2}\right)$ and carbon dioxide $\left(\mathrm{PtCCO}_{2}\right)$ tensions, which directly measure skin oxygenation and $\mathrm{CO}_{2}$ retention, may also be used to objectively evaluate skin oxygenation and perfusion in trauma patients beginning with initial resuscitation. A preliminary study in 48 trauma patients documented that, compared with survivors, patients who died had significantly lower $\mathrm{PtcO}_{2}$ and higher $\mathrm{PtcCO}_{2}$ values beginning with the early stage of resuscitation. All patients who maintained $\mathrm{PtcO}_{2}>150$ torr $(19.99 \mathrm{kPa})$ throughout monitoring survived. Periods of $\mathrm{PtcO}_{2}<50$ torr $(6.66 \mathrm{kPa})$ for $>60$ min or $\mathrm{PtcCO}_{2}>60$ torr $(8.00 \mathrm{kPa})$ for $>30$ min were associated with $90 \%$ mortality and $100 \%$ morbidity. These authors concluded that $\mathrm{PtcO}_{2}$ and $\mathrm{PtcCO}_{2}$ monitoring continuously evaluated tissue perfusion and served as an early warning in critically injured patients during resuscitation immediately after hospital admission. ${ }^{48}$ 


\section{When to Transfuse?}

The treatment of hemorrhagic shock with blood transfusion is appropriate in trauma for ongoing significant hemorrhage and when the patient is unresponsive to crystalloid resuscitation and manifesting physiologic signs of persistent shock (hypotension, tachycardia, oliguria, lactic acidosis), indicating that oxygen consumption is dependent on hemoglobin concentration (critical oxygen delivery). Transfusion of blood, platelets and plasma is of course necessary in the treatment of acute hemorrhagic shock with its associated complications of dilutional coagulopathy and thrombyocytopenia, since the primary goal is control of hemorrhage, restoration of blood volume, and normalization of coagulation. Concomitant attempts at prompt cessation of hemorrhage are also necessary.

A number of studies, however, have documented that blood transfusion is associated with adverse outcome in trauma patients, including multiple organ failure, systemic inflammation, increased infectious complications, increased incidence of pneumonia and pulmonary complications, and long-term microchimerism. ${ }^{49,50}$ Therefore, once hemorrhage control has been established in acute trauma, we should attempt to minimize the use of blood transfusion for the treatment of asymptomatic anemia in trauma patients.

It has been clearly documented in a prospective randomized clinical trial (Transfusion Requirements in Critical Care Trial, TRICC) that a restrictive transfusion strategy (blood transfusion only if hemoglobin $<7 \mathrm{~g} / \mathrm{dL}$ ) yielded equivalent outcomes compared to a liberal transfusion strategy (blood transfusion if hemoglobin $<10 \mathrm{~g} / \mathrm{dL}$ ) in critically ill patients with anemia. ${ }^{51}$ Mortality rates (30-day) were similar in the two groups (18.7\% vs. $23.3 \% ; p=0.11$ ). However, mortality was significantly lower in the restrictive transfusion strategy among patients who were less acutely ill (those with an Acute Physiology and Chronic Health Evaluation II score $\leq 20$ (8.7\% in the restrictive-strategy group and $16.1 \%$ in the liberal-strategy group; $p=0.03$ ) and among patients who were less than 55 years of age $(5.7 \%$ and $13.0 \%$, respectively; $p=0.02$ ). A recent post-hoc cohort analysis of the trauma patients $(n=203)$ in the TRICC trial concluded that a restrictive red blood cell transfusion strategy was safe for critically ill multiple-trauma patients. ${ }^{52}$

In fact, recent studies suggest that increasing hemoglobin with the transfusion of aged stored blood does not improve perfusion of the microcirculation..$^{53}$ Lack of efficacy of red blood cell transfusion is likely related to storage time, increased endothelial adherence of stored red blood cells, nitric oxide binding by free hemoglobin in stored blood, ${ }^{54}$ donor leukocytes, host inflammatory response, and reduced red cell deformability. A recent animal study documented that improving the microcirculation by other measures is more effective than transfusion of red blood cells to correct metabolic disorder in experimental hemorrhagic shock. ${ }^{55}$ Therefore, all efforts to restrict red blood cell transfusion in trauma patients once the patient has completed resuscitation from hemorrhagic shock is advisable.

\section{Conclusion}

It has now been recognized that resuscitation research will require a national coordinated effort of prospective randomized clinical trials in order to ascertain the answers to these difficult questions in trauma resuscitation, including identification of the ideal resuscitative fluid and appropriate resuscitation endpoints in trauma. The PULSE (Post Resuscitative and Initial Utility of Life Saving Efforts) Trauma Work Group published their recommendations for a national coordinated trauma research program to conduct such studies for the future. ${ }^{56}$ The Resuscitation Outcomes Consortium was recently established, with a mission to conduct multicenter clinical trials and outcomes research in trauma.

\section{Key points}

1. Moderate fluid resuscitation with isotonic crystalloid is recommended for most trauma victims.

2. Standard hemodynamic parameters (heart rate, blood pressure) do not adequately quantify the degree of hemorrhagic shock and physiologic derangement in trauma patients.

3. Lactate and base deficit can be used to identify trauma patients that are at risk of adverse outcome and require ongoing fluid resuscitation.

4. No absolute resuscitation endpoints are universally accepted in trauma.

5. Patients who achieve supranormal oxygen delivery parameters have improved survival in trauma. 
1. Heckbert SR, Vedder NB, Hoffman W, et al. O utcome after hemorrhagic shock in trauma patients. J Trauma 1998:45:545-9.

2. Porter JM, Ivatury RR. In search of the optimal end points of resuscitation in trauma patients: a review. J Trauma 1998:44:908-14.

3. Dabrowski GP, Steinberg SM, Ferrara JJ, Flint LM. A critical assessment of endpoints of shock resuscitation. Surg Clin N orth Am 2000;80:825-44.

4. Revell M, G reaves I, Porter K. Endpoints for fluid resuscitation in hemorrhagic shock. J Trauma 2003;54:S63-7.

5. Holmes JF, Sakles JC, Lewis G, Wisner DH. Effects of delaying fluid resuscitation on an injury to the systemic arterial vasculature. Acad Emerg M ed 2002;9:267-74.

6. Burris D, Rhee P, Kaufmann C, et al. Controlled resuscitation for uncontrolled hemorrhagic shock. J Trauma 1998;46:216-23.

7. Capone A, Safar P, Stezoski SW, Peitzman A, Tisherman S. Uncontrolled hemorrhagic shock outcome model in rats. Resuscitation. 1995 Apr;29(2):143-52.

8. Capone AC, Safar P, Stezoski W, Tisherman S, Peitzman AB. Improved outcome with fluid restriction in treatment of uncontrolled hemorrhagic shock. J Am Coll Surg 1995;180:49-56.

9. Kim SH, Stezoski SW, Safar P, C apone A, Tisherman S. Hypothermia and minimal fluid resuscitation increase survival after uncontrolled hemorrhagic shock in rats. J Trauma 1997;42:213-22.

10. Leppaniemi A, Soltero R, Burris D, et al. Fluid resuscitation in a model of uncontrolled hemorrhage: too much too early, or too little too late? J Surg Res 1996;63:413-8.

11. Matsuoka T, Hildreth J, Wisner DH. Uncontrolled hemorrhage from parenchymal injury: is resuscitation helpful? I Trauma 1996;40:915-21; discussion 921-2.

12. Xiao N, Wang XC, Diao YF, Liu R, Tian KL, Effect of initia fluid resuscitation on subsequent treatment in uncontrolled hemorrhagic shock in rats. Shock 2004;21:276-80.

13. Bickell WH, Wall MJ Jr, Pepe PE, et al. Immediate versus delayed fluid resuscitation for hypotensive patients with penetrating torso injuries. N Engl J M ed 1994;331:1105-9.

14. Turner J, Nicholl J, Webber L, C ox H, Dixon S, Yates D. A randomized controlled trial of prehospital intravenous fluid replacement therapy in serious trauma. Health Technol Assess 2000;4:1-57.

15. Dula DJ, Wood GC, Rejmer AR, Starr M, Leicht M. Use of prehospital fluids in hypotensive blunt trauma patients. Prehosp Emerg Care 2002;6:417-20.

16. G reaves I, Porter KM, Revell MP. Fluid resuscitation in prehospital trauma care: a consensus view. J R Coll Surg Edinb 2002;47:451-7

17. Rhee P, Koujstova E, Alam HB. Searching for the optimal resuscitation method: Recommendations for the initial fluid resuscitation of combat casualties. J Trauma 2003;54:552-62.

18. Stern SA. Low-volume fluid resuscitation for presumed hemorrhagic shock: helpful or harmful? Curr Opin Crit Care 2001;7:422-30.

19. Dutton RP, Mackenzie CF, Scalea TM. Hypotensive resuscitation during active hemorrhage: impact on in-hospital mortality. J Trauma 2002;52:1141-6.

20. McN elis J, Marini $C P$, Jurkiewicz $A$, et al. Prolonged lactate clearance is associated with increased mortality in the surgical intensive care unit. Am J Surg 2001;182:481-5.

21. Husain FA, Martin MJ, Mullenix PS, Steele SR, Elliott DC. Serum lactate and base deficit as predictors of mortality and morbidity. Am J Surg 2003185:485-91
22. Tisherman SA, Barie P, Bokhari F, et al. Clinical Practice Guideline: Endpoints of Resuscitation. Eastern Association for the Surgery of Trauma. Available at: www.east.org.

23. Bishop MH, Shoemaker WC, Appel PL, et al. Prospective, randomized trial of survivor values of cardiac index, oxygen delivery, and oxygen consumption as resuscitation endpoints in severe trauma. J Trauma 1995;38:780-7

24. Durham RM, Neunaber K, Mazuski JE, Shapiro MJ, Baue AE. The use of oxygen consumption and delivery as endpoints for resuscitation in critically ill patients. J Trauma 1996;41:32-9 discussion 39-40.

25. Velmahos GC, Demetriades D, Shoemaker WC, et al Endpoints of resuscitation of critically injured patients: norma or supranormal? A prospective randomized trial. Ann Surg 2000;232:409-18

26. Kern JW, Shoemaker WC. Meta-analysis of hemodynamic optimization in high-risk patients. Crit Care M ed 2002;30:1686-92.

27. McKinley BA, Kozar RA, C ocanour C S, et al. Normal versus supranormal oxygen delivery goals in shock resuscitation: the response is the same. J Trauma 2002;53:825-32.

28. Taylor MD, Tracy JK, Meyer W, Pasquale M, Napolitano LM. Trauma in the elderly: intensive care unit resource use and outcome. J Trauma 2002;53:407-14.

29. Schulman AM, Claridge JA, Young JS. Young versus old factors affecting mortality after blunt traumatic injury. Am Surg 2002;68:942-7; discussion 947-8.

30. Gattinoni L, Brazzi L, Pelosi P, et al. A trial of goal-oriented hemodynamic therapy in critically ill patients. $\mathrm{SvO}_{2}$ Collaborative Group. N Engl J Med 1995;333:1025-32.

31. Committee on Fluid Resuscitation for $C$ ombat Casualties. Fluid Resuscitation: State of the Science for Treating Combat Casualties and Civilian Injuries. Report of the Institute of Medicine. Washington D.C.: National Academy Press: June 1, 1999. Available at: www.iom.edu.

32. Wade CE, Grady JJ, Kramer GC. Efficacy of hypertonic saline dextran fluid resuscitation for patients with hypotension from penetrating trauma. J Trauma 2003;54(5 Suppl):S144-8.

33. Cooper DJ, Myles PS, McDermott FT, et al. Prehospita hypertonic saline resuscitation of patients with hypotension and severe traumatic brain injury: a randomized controlled trial. JAMA 2004;291:1350-7.

34. Dieterich HJ. Recent developments in European colloid solutions J Trauma 2003;54(5 Suppl):S26-30

35. Lang K, Suttner S, Boldt J, Kumle B, Nagel D. Volume replacement with HES 130/0.4 may reduce the inflammatory response in patients undergoing major abdominal surgery. Can J Anaesth 2003;50:1009-16

36. Boldt J, Ducke M, Kumle B, Papsdorf M, Zurmeyer EL. Influence of different volume replacement strategies on inflammation and endothelial activation in the elderly undergoing major abdominal surgery. Intensive C are M ed 2004;30:416-22.

37. Knudson MM, LeeS, Erickson V, Morabito D, Derugin N, Manley GT. Tissue oxygen monitoring during hemorrhagic shock and resuscitation: a comparison of lactated Ringer's solution, hypertonic saline dextran, and HBOC-201. J Trauma 2003;54:242-52.

38. C hang MC, Miller PR, D'Agostino R Jr, Meredith JW. Effects of abdominal decompression on cardiopulmonary function and visceral perfusion in patients with intra-abdominal hypertension J Trauma 1998;44:440-5

39. Marr AB, Moore FA, Sailors RM, et al. Preload optimization using "Starling Curve" generation during shock resuscitation: Can it be done? Shock 2004;21:300-5.
40. Kincaid EH, Meredith JW, C hang MC. Determining optima cardiac preload during resuscitation using measurements of ventricular compliance. J Trauma 2001;50:665-9.

41. Chang MC, Meredith JW, Kincaid EH, Miller PR. Maintaining survivors' values of left ventricular power output during shock resuscitation: a prospective pilot study. J Trauma 2000;49:26 33; discussion 34-7.

42. Beilman GJ, Broehler KE, Lazaron V, Ortner JP. Near-infrared spectroscopy measurement of regional tissue oxyhemoglobin saturation during hemorrhagic shock. Shock 1999;12:196-200.

43. Crookes BA, Cohn SM, Burton EA, Nelson J, Proctor KG $N$ oninvasive muscle oxygenation to guide fluid resuscitation after traumatic shock. Surgery 2004;135:662-70.

44. Chaisson NF, Kirschner RA, Deyo DJ, Lopez JA, Prough DS Kramer GC. Near-infrared spectroscopy-guided closed-100 resuscitation of hemorrhage. J Trauma 2003;54(5 Suppl):S183-92.

45. Crookes B, C ohn S, Bloch S, et al. Can muscle near infrared spectroscopy identify the severity of shock in trauma patients? Presented at the Eastern Association for the Surgery of Trauma Seventeenth Scientific Assembly, January 14-17, 2004, Amelia Island, Florida.

46. Velmahos GC, Wo C C, Demetriades D, Shoemaker WC. Early continuous noninvasive haemodynamic monitoring afte severe blunt trauma. Injury 1999;30:209-14

47. C ottis R, Magee $\mathrm{N}$, Higgins DJ. Haemodynamic monitoring with pulse-induced contour cardiac output ( $\mathrm{PiCCO}$ ) in critica care. Intensive Crit Care Nurs 2003;19:301-7.

48. Tatevossian RG, Wo CC, Velmahos GC, Demetriades D Shoemaker WC. Transcutaneous oxygen and $\mathrm{CO} 2$ as early warning of tissue hypoxia and hemodynamic shock in critically ill emergency patients. Crit Care M ed 2000;28:2248-53.

49. Silliman CC, Moore EE, Johnson JL, G onzalez FJ, Biffl WL. Transfusion of the injured patient: Proceed with caution. Shock 2004;21:291-9.

50. Malone DL, Dunne J, Tracy JK, Putnam AT, Scalea TM, Napolitano LM. Blood transfusion, independent of shock severity, is associated with worse outcome in trauma. J Trauma 2003:54:898-905; discussion 905-7.

51. Hebert PC, Wells G, Blajchman MA, et al. A multicenter randomized, controlled clinical trial of transfusion requirements in critical care. Transfusion Requirements in Critical Care Investigators, Canadian Critical Care Trials Group. N Engl J Med 1999;340:409-17

52. McIntyre L, Hebert PC, Wells G, et al. Is a restrictive transfusion strategy safe for resuscitated and critically ill trauma patients? J Trauma 2004;57:563-8; discussion 568.

53. N apolitano LM, Corwin HL. Efficacy of red blood cell transfusion in the critically ill. Crit Care Clin 2004;20:255-68.

54. Schechter AN, Gladwin MT. Hemoglobin and the paracrine and endocrine functions of nitric oxide. Clinical implications of basic research. N Engl J M ed 2003;348:1483-5.

55. Wettstein R, Tsai AG, Erni D, Lukyanov AN, Torchilin VP, Intaglietta M. Improving microcirculation is more ffective than substitution of red blood cells to correct metabolic disorder in experimental hemorrhagic shock. Shock 2004;21:235-40.

56. C arrico CJ, Holcomb JB, C haudry IH; PULSE Trauma Work Group (Post Resuscitative and Initial Utility of Life Saving Efforts). Scientific priorities and strategic planning for resuscitation research and life saving therapy following traumatic injury: report of the PULSE Trauma Work Group. Post Resuscitative and Initial Utility of Life Saving Efforts. Shock 2002;17:165-8. 